Running Head: BI-FACTOR ROTATION BASED ON LOADING DIFFERENCES

Improving Bi-factor Exploratory Modelling: Empirical Target Rotation Based on Loading Differences

Garcia-Garzon, Eduardo.

Universidad Autónoma de Madrid (Spain)

Abad, Francisco J.

Universidad Autónoma de Madrid (Spain)

Garrido, Luis E.

Pontificia Universidad Católica Madre y Maestra (Dominican Republic)

This manuscript has been accepted for publication in

Methodology.

https://doi.org/10.1027/1614-2241/a000163

Corresponding Author:

Eduardo Garcia-Garzon.

Departamento de Psicología Social y Metodología

Facultad de Psicología

Universidad Autónoma de Madrid.

Ciudad Universitaria de Cantoblanco,

28049 Madrid (Spain)

Email: eduardo.garciag@uam.es 


\begin{abstract}
Bi-factor exploratory modelling has recently emerged as a promising approach to multidimensional psychological measurement. However, state-of-the-art methods relying on target rotation require researchers to select an arbitrary cut-off for defining the target matrix. Unfortunately, the consequences of such choice on factor recovery remain uninvestigated under realistic conditions (e.g., factors differing in their average loadings). Built upon the iterative target rotation based on Schmid-Leiman algorithm (SLi), a novel method is here introduced (SLiD). SLiD settles an empirical, factorspecific cut-off based on the first prominent one-lagged difference of sorted squared normalized factor loadings. SLiD and SLi with arbitrary cut-offs (ranging from .05 to .20) performance was evaluated via Monte-Carlo simulation manipulating sample size, number of specific factors, number of indicators and cross-loading magnitude. Results indicate that SLiD performed the best for all conditions. For SLi, and due to the presence of minor factors, smaller cut-offs (i.e., .05) outperformed higher ones (i.e., $.20)$.
\end{abstract}

Keywords: bifactor; target rotation; Schmid-Leiman; exploratory factor analysis. 


\section{Improving Bi-factor Exploratory Modelling: Empirical Target Rotation Based on Loading Differences}

Bi-factor models (Holzinger \& Swineford, 1937) are regarded today as crucial tools for evaluating psychological constructs, as they allow for the inspection of the simultaneous direct influence of a general and specific factors over a set of indicators (Reise, 2012). Although traditionally studied by means of confirmatory models that conform to a simplest solution for the specific factors, the suitability of such proposals has been recently questioned (Morin, Arens \& Marsh, 2015). This has led to a growing interest on more realistic exploratory bi-factor models, where each indicator is expected to be potentially explained by all factors (Abad, Garcia-Garzon, Garrido, \& Barrada, 2017; Mansolf \& Reise, 2016; Waller, 2017).

Automated bi-factor target methods have emerged as a compelling alternative for performing exploratory bi-factor analysis (Abad et al., 2017). However, these procedures require researchers to choose a single cut-off to distinguish which factor loadings are expected to be negligible in the final rotated solution (Browne, 1972).Unfortunately, the impact of this decision has not been sufficiently examined (Myers, Ahn, \& Jin, 2013; Myers, Jin, Ahn, Celimli, \& Zopluoglu, 2015). Furthermore, as the application of a single cut-off could be inappropriate, some authors have suggested the use of factor-specific cut-offs for achieving simple solutions (Trendafilov, 1994; Lorenzo-Seva, 1999).

The current study aims to propose a new method for target specification based on factor loading differences to empirically determine factor-specific cut-offs. This new method is applied to the Schmid-Leiman with iterative target rotation (SLi; Abad et al., 2017). SLi is a bi-factor rotation algorithm that has been shown to perform more accurately than both, analytical bi-factor rotations and non-iterative Schmid-Leiman 
target rotation (Abad et al., 2017). The performance of the proposed method, hereafter $\mathrm{SLiD}$, is therefore tested against that of SLi with a range of predetermined fixed cutoffs.

The rest of the article is structured as follows. First, the Schmid-Leiman orthogonalization and the automatic specification of target matrices are briefly reviewed. Next, an overview regarding the iterative target rotation procedure is presented. In the following section, an introduction to empirical bi-factor target rotation based on loading differences method is depicted. Subsequently, the results from an extensive Monte Carlo simulation comparing the accuracy of SLiD to that of SLi with arbitrary cut-offs are examined. Finally, implications for future research are discussed.

In the remainder of the article, a factor pattern matrix is said to follow a bi-factor model if and only if a general factor (i.e., which directly influences most indicators) along with several specific factors (i.e., which influence distinct groups of indicators within the same set) are present, where the latter explains variance residual to the former, and all factors are orthogonal (Holzinger \& Swineford, 1937; Reise, 2012). Noteworthy, an item is always expected to load in the general and in an unrestricted number of specific factors. Therefore, cross-loadings arising from an item reflecting variance from several group factors are to be expected (Morin, Arens \& Marsh, 2015), a situation that constitutes a deviation from traditional, simple bi-factor structures.

\section{The Schmid-Leiman Transformation}

Early proposals for conducting exploratory bi-factor analysis relied on the Schmid-Leiman orthogonalization (SL; Schmid \& Leiman, 1957), which transforms a hierarchical solution into a model with the appearance of a bi-factor model. However, the latter model presents linear dependencies between the general and specific factors 
loadings (Mansolf \& Reise, 2016). Indeed, Waller (2017) demonstrates that for any structure defined by three (or more) common factors, an SL solution is not unique (p.1), and it represents a low-rank solution when compared with a bi-factor structure without such linear constraints ${ }^{1}$. Consequently, a SL solution would only provide unbiased estimates when recovering a true structure presenting such constraints (namely, a hierarchical model; Abad et al., 2017; Reise, Moore, \& Maydeu-Olivares, 2011). Unfortunately, SL solutions are often considered as full-rank bi-factor structures, and misguidedly applied to compute statistics such as scale reliability. In Waller's (2017) words:

"the [SL] model sometimes fools researchers into believing that the SL transformation yields a higher-dimensional representation of the lower-order, correlated model. This belief is false" (p.4).

Two distinct classes of methods for estimating exploratory bi-factor structures have been proposed. Firstly, Jennrich \& Bentler $(2011,2012)$ formulated the direct analytical rotations based on the quartimin and geomin rotations. Unfortunately, such procedures have been proved to be either unreliable for recovering complex structures (bi-quartimin), or prone to present local minima and factor collapse problems (bigeomin; Hattori et al., 2017, Mansolf \& Reise, 2016). Secondly, the rediscovery of the target rotation as an alternative for recovering complex structures has prompted the emergence of bi-factor exploratory methods based on this rotation.

\section{Automatic Specification of Target Matrices}

A target rotation (also called "Procrustes rotation") is a semi-confirmatory procedure intended to approximate a rotated solution towards a pre-defined factor

${ }^{1}$ The term "bi-factor is restricted hereafter to models not presenting the aforementioned constraints between general and specific factor loadings. 
pattern. To do so, a target matrix (B), with same dimensions as the factor loading matrix $(\boldsymbol{\Lambda})$, must be defined as follows: If the value of an entry is expected to be negligible in the targeted rotated solution, that entry is fixed as zero in B. Otherwise, the entry is left unspecified (partially specified target rotation; Browne, 1972). Afterwards, the target rotation is performed by minimizing the following loss function:

$$
f(L)=\sum_{j=1}^{m} \sum_{i \in I_{j}}\left(\lambda_{i j}-b_{i j}\right)^{2}
$$

Where $\lambda_{i j}$ is a loading in the $\mathrm{i}_{\text {th }}$ row and $\mathrm{j}_{\text {th }}$ column of $\boldsymbol{\Lambda}, b_{i j}$ is an entry of the $\mathrm{i}_{\text {th }}$ row and $j_{\text {th }}$ column of $\mathbf{B}$, and $I$ represents the subscript for fixed target loadings.

Although researchers can manually specify $\mathbf{B}$ according to their theoretical expectations, modern approaches rely on the automatic detection of which loadings should fixed as zero in B. Reise, Moore, \& Maydeu-Olivares (2011) firstly advocated the use of automated bi-factor target rotations. They argued that even though solutions obtained by means of a Schmid-Leiman transformation result in biased estimates, they could provide the basis for accurately defining partially specified target matrices. This procedure led to the adequate recovery of exploratory bi-factor models, especially when cross-loadings were present in the structure.

\section{Iteration of Partially Specified Bi-factor Target Matrices}

Moore et al. (2015) demonstrated that an iterative target rotation procedure, where each rotated target solution formed the basis for defining a new target matrix, led to improved target specification, and that such refinement directly translated into enhanced rotation accuracy. Abad et al. (2017) showed in an extensive simulation that SLi, which combines an iterative target rotation with Reise's et al. (2011) procedure, outperformed the original non-iterative method as well as both direct analytical bi-factor rotations. 
Unfortunately, all automated target methods hitherto presented request researchers to decide a single cut-off for separating expected substantive and negligible factor loadings. While recommendations for selecting such a threshold do exist for the general exploratory factor analysis case (between .30 and .50; Izquierdo, Olea \& Abad, 2014), the impact of this choice on rotation performance has been overlooked in the literature. Ultimately, theoretical recommendations could be misleading in a given factor analysis, as a suitable cut-off point may be dependent on the specific conditions of the factor model estimation (i.e., sample size) or could vary across factors when these differ in their factor loading distributions. Therefore, a set of cut-offs would yield a correct targeted rotated solution if, and only if, they correctly identify, for each specific factor separately, all, and exclusively, the near-zero loadings in the targeted rotated matrix (i.e., minimizes target rotation criterion; Kiers, 1994). Consequently, a single, fixed cutoff point would result in misspecification errors when the strength of the involved factors varies, and the structure departs from a simple bi-factor model. The study of such conditions (illustrated below) demonstrated that cut-offs shall be empirically estimated rather than arbitrary chosen.

\section{Empirical Bi-factor Target Rotation Based on Loading Differences}

Under a bi-factor solution where all specific factors follow the simplest structure case, appropriate cut-off points that lead to a correct target definition might be easily found. If the model is properly estimated (i.e., adequate sample size), each specific factor yields a factor loading distribution with two distinctive groups: a first set of loadings near zero and another encompassing substantive loadings. Hence, such appropriate cut-offs will correctly fix to zero in the target matrix all near-zero loadings. 
To ensure better separation between near-zero and substantive loadings, all factor loadings are traditionally transformed by means of Kaiser's normalization. This procedure consists in multiplying each $\Lambda$ row by the inverse of the square root of the correspondent row communality. Normalization is also usually applied in this field to avoid the fact that large rows of $\boldsymbol{\Lambda}$ have more influence in the rotation criteria than smaller rows (Jennrich, 2004). Additionally, and to further increase substantive factor loadings saliency, normalized loadings can be squared (Lorenzo-Seva, 1999). After applying such transformations, the size of each squared normalized factor loading becomes a function of the item complexity (i.e., number and magnitude of crossloadings) and, for complex items, of its primariness (i.e., if the substantive loading is the primary or a secondary loading). For instance, for items following a simple population bi-factor structure, the matrix of squared row-normalized loadings should be composed by zero and one entries, which would facilitate setting a correct cut-off. A detailed discussion of the effects of such transformations can be found in Lorenzo-Seva (1999) and Browne (2001).

The presence of small cross-loadings in one specific factor, which is always to be expected (Asparouhov \& Muthén, 2009; Morin, Arens \& Marsh, 2015), introduces a third group of items whose (squared normalized) factor loadings are somewhere situated between the near-zero (i.e., for items non-loading on the factor) and near-one loadings (i.e., for factorially simple items only loading in the factor). To be bear in mind that if, by definition (Eq.1), the rotation criterion only depends on the specified elements of the target matrix, wrongly fixing any potential minor cross-loading in the target matrix will result in impaired target rotation. Accordingly, an appropriate cut-off for each specific factor should result in such cross-loadings also being freed in the target matrix. Therefore, a statistical criterion to define which (squared normalized) factor loadings 
constitute meaningful specific loadings is in need. Additionally, the impact of crossloadings on cut-off estimation is ameliorated -or worsened- depending on factor loading primariness. Structures presenting low magnitude factor loadings will result in less distinctive groups of near-zero and substantive factor loadings, ultimately hampering cut-off points identification.

Thus, under conditions where cross-loadings and low average loadings factor are expected, it is implausible that a single, fixed cut-off point would correctly identify all elements in the target matrix. Any method applying fixed cut-offs (i.e., SLi) is therefore anticipated to commit many misspecification errors, and to result in a biased factor solution. To overcome such limitations, a novel algorithm based on empirical, factorspecific cut-offs is here introduced: SLiD. This method, which is regarded as an improvement of the SLi algorithm as it introduces an empirical cut-off estimation within that algorithm, is likely to limit misspecification errors in target matrix definition, and to result in improved factor recovery. To illustrate SLiD functioning, an analysis of a popular bi-factor structure presenting the aforementioned characteristics (Abad et al., 2017; Chen, West \& Souza; 2006) is presented (Table 1).

\section{[PLEASE INSERT TABLE 1 HERE]}

The proposed SLiD method estimates factor-specific loading cut-offs based on the distribution of the differences between the sorted squared normalized factor loadings. To find this empirical cut-off, SLiD performs several steps aimed to separate near-zero loadings to be fixed in the target matrix, and meaningful cross-loadings to be freed (note that general factor loadings are always freed, so they are not evaluated when estimating cut-offs). 
Starting from a standardized SL solution (Panel A, Table 1), the squared normalized loadings are computed (Panel B, Table 1). Secondly squared normalized loadings are sorted per factor to detect a "jump" in the sequence (Panel C, Table 1). A similar strategy was deployed by Jennrich (2004) to detect the number of near-zero values to be fixed in a target matrix in a Simplimax rotation (Kiers, 1994) by visually detecting the first meaningful "jump" in a SAL plot (Sorted Absolute Loadings Plots; Figure 5; Jennrich, 2004). Thirdly, one-lagged differences are computed (differences between a factor loading and its immediate predecessor; Panel D, Table 1). For each specific factor (e.g., SF1), small one-lagged differences are expected for blocks of homogenous items. For instance, for SF1 close-to-zero one-lagged differences are found for the group of non-loading items (i.e., 11, 8, 12, 9, 10, 15, 17, 6, 7, and 16) and for almost all the group of simple items (i.e., 1, 3, 4, and 5). In contrast, the higher onelagged differences represent some "jumps" in the distribution of loadings (i.e., .16, .43, and .37). The first "jump" reveals a potential candidate for representing the separation of the last near-zero loading (i.e., .02) and the first substantive loading (i.e., .18).

We propose the average lagged difference as criterion to identify which items represent the boundary between such groups (in italics, last row in Panel D, Table 1; e.g., for SF1, .06), setting the first one-lagged difference in the sorted distribution with a value higher than the criterion as the first "jump" (bolded an underlined values in Panel D, Table 1; e.g., for SF1, .16). The rationale behind is that the number of "jumps" should be necessarily a small proportion of the one-lagged differences. Lastly, for each factor, the cut-off is settled to the factor loading corresponding to the lower endpoint of the interval represented by this difference (Panel E, Table 1; e.g., for SF1, item.11). In Table 1 example, when compared previously applied cut-offs such as .15 (Reise, Moore 
\& Maydeu-Olivares, 2011) or .20 (Abad et al., 2017), SLiD found three additional small cross-loadings to be additionally freed in the target matrix (Panel E, Table 1).

It is anticipated that the SLiD method will find, for each specific factor, an accurate cut-off regardless of the distribution of the loading differences across factors. Unfortunately, SLiD would not produce a satisfactory solution if a non-identified solution is to be produced (e.g., if at least $(j-1) / 2$ targets in each column are not fixed; Asparouhov \& Muthén (2009); condition C1 to C3 adapted to orthogonal structures in Peeters; 2012). Therefore, SLiD evaluates if Peeters (2012) conditions C1 to C3 are met in the target matrix (in a similar fashion to Mplus target rotation; Asparouhov \& Muthén, 2009; Myers, Ahn, \& Jin, 2013). If any of such conditions are not met, the smallest non-fixed loading of the sorted normalized factor loading distribution would be fixed in the target rotation. Due to space constrains, readers are remitted to original sources for a more detailed explanation of mentioned factor identification conditions.

\section{Algorithm 1}

Schmid-Leiman Iterative Bi-factor Difference-based Target Rotation (SLiD)

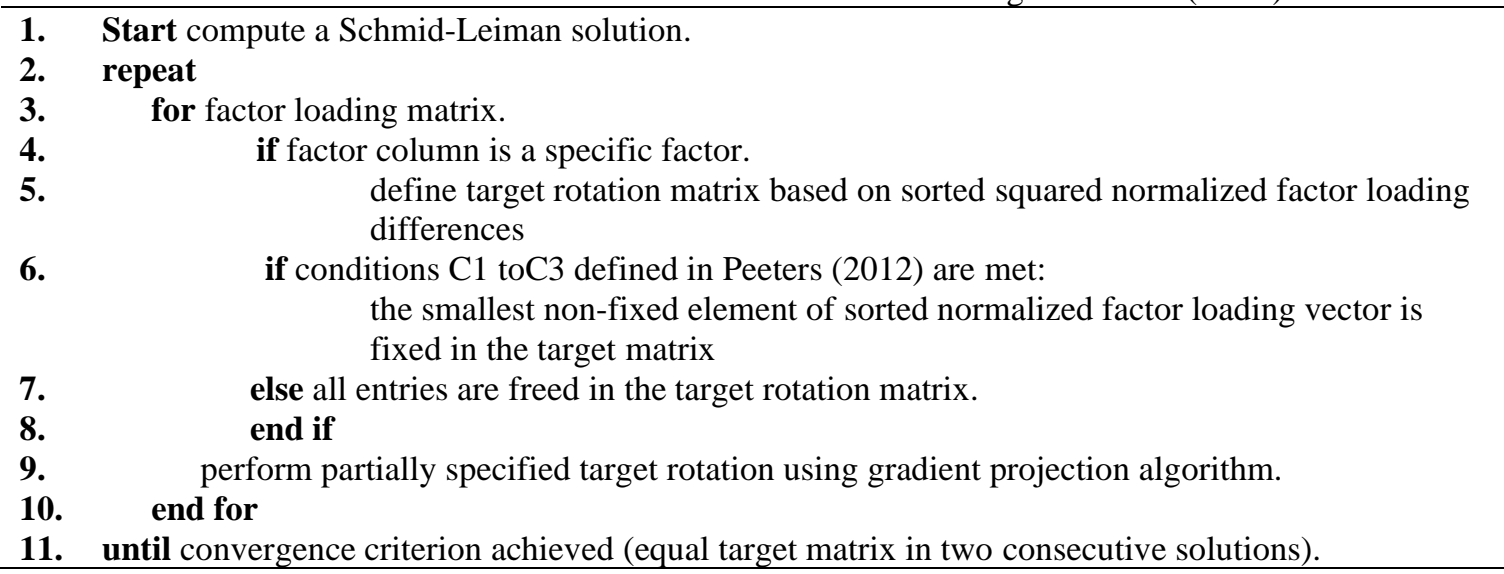

To conclude, the objective of this article is three-folded: First, to investigate for the first time the effect of applying arbitrary cut-offs on bi-factor target rotation. Second, to understand the properties of the new proposed algorithm for exploratory bi- 
factor modelling (SLiD). Third, to compare the performance of SLiD and SLi under a set of realistic conditions by means of a Monte Carlo simulation.

\section{Method}

\section{Simulation Design}

A Monte Carlo study was designed to compare SLiD against the SLi target rotation using arbitrary cut-offs. For SLi, four cut-off points were examined (.05, .10, .15 , and .20 ), covering the range previously considered in bi-factor exploratory research (Abad et al., 2017; Reise, Moore, \& Haviland, 2010; Waller, 2017). Each rotation is hereafter referred to as SLi followed by the applied cut-off point.

Several variables were manipulated in the following Monte Carlo simulation (Table 2): sample size (N), number of variables per specific factor (VAR.SF), number of specific factors (NUM.SF), and cross-loading size (CROSS.SD), yielding $3 \times 3 \times 2 \times 3=$ 54 conditions fully crossed. Population bi-factor structures were simulated as follows: a) general factor loadings ranged from .575 to.625 in equal increments and were randomly sorted; b) for each structure, specific factors were simulated varying in the average loading size (high, medium and low); specifically, loadings ranged in equal increments from .575 to.625 (first or first two factors, depending upon NUM.SF), from .425 to .475 (central or two central factors) and from .275 to .325 (last or last two factors).

For each structure, the first two items in each factor were set as markers (all their cross-loadings were fixed to zero). For the remaining indicators, cross-loadings were drawn from a normal distribution with a mean of 0 and a standard deviation of .05 (low condition: $95 \%$ of cross-loadings ranged between \pm .098 ), .10 (medium condition: $95 \%$ of cross-loadings ranged between \pm .196 ) and .15 (high condition: $95 \%$ of cross-loadings 
ranged between \pm .294 ). This procedure, also found in Meade (2008), simulated crossloadings distributions that are consistent with those commonly found in the literature (Bollmann, Heene, \& Küchenhoff, 2015) . If either the population correlation matrix was not positive semi-definite (i.e., minimum eigenvalue $\leq 0$ ) or the item communalities were higher than .90 , all simulated cross-loadings were replaced. Simulated average item communalities across conditions depended upon number of factors simulated and cross-loading size, ranging from .580 (i.e., NUM.SF $=3$; CROSS.SD $=.05$ ) to .732 (i.e., NUM.SF = 6; CROSS.SD = .15).

[PLEASE INSERT TABLE 2 HERE]

\section{Data Generation}

A total of 800 sample data matrices were simulated for each condition. First, for each replication the cross-loadings were generated. Then, the population correlation matrix, obtained by inserting unities in the diagonal of the reproduced correlation matrix, was used to generate a matrix of random standard normal observed variables with dimensions of sample size $(\mathrm{N})$ per number of specific variables (VAR.SF) with the function rmvnorm (mvnorm package; Genz et al., 2017; R Development Core Team, 2017).

\section{Rotation Methods}

Scripts in R (R Development Core Team, 2017) were applied to obtain the rotated solutions. The SLi solutions were computed using the SLi function in Abad et al. (2017), while the SLiD code is provided in the Electronic Supplementary Material 1. In all the cases, the unweighted least squares estimator was used for factor extraction (psych package; Revelle, 2016).

\section{Accuracy Criteria}


Accuracy was assessed by means of Tucker's congruence coefficient (c.c.; Tucker, 1951), which measures similarity between two vectors. The c.c. values are bounded between -1 and 1, where values ranging from .85 to .94 are considered as fair and values over .95 reflect that the two factors should be considered equal (LorenzoSeva \& ten Berge, 2006).

To accurately compute c.c. values, estimated factors must be accordingly aligned with their correspondent population factor. Firstly, an estimated factor was reverted if the sum of factor loadings was negative. Secondly, c.c. was computed for every possible factor permutation, retaining the solution that minimized the average deviation between that solution and the population matrix.

To compare c.c. means across conditions, analyses of variance were performed using the jamovi program 0.8.1.13 (jamovi project, 2018). Partial eta squared $\left(\eta_{p}^{2}\right)$ effect sizes were reported, where $\eta_{p}^{2}>.01, \eta_{p}^{2}>.06$, and $\eta_{p}^{2}>.14$ were considered as small, medium, and large effects, respectively (Cohen, 1988). Additionally, to depict how each distinctive factor was recovered (i.e., general factor, and specific factors with high, medium, and low average factor loadings), the percentage of samples across conditions showing fair (i.e., c.c. $\geq .85$ ) and good (i.e., c.c. $\geq .95$ ) recovery were computed for the different rotation methods.

\section{Results}

Mean c.c. for each rotation procedure and simulated condition are depicted in Table 3. Firstly, SLiD was the preferred method in all conditions, consistently producing the highest c.c. for all levels considered. Secondly, SLi with a fixed cut-off point showed an inadequate overall performance, with mean c.c. values decreasing as cut-offs increased. In this line, it is noteworthy that SLi.20 only reached a fair recovery 
(c.c. $\geq .85$ ) for one marginal condition (c.c. $=.887$ with low cross-loadings). In general, for all methods increasing either the sample size or the number of variables per specific factor resulted in increased factor recovery (except for SLi.20), while increasing the number of factors or the cross-loading size decreased their accuracy.

\section{[PLEASE INSERT TABLE 3 HERE]}

Table 4 provides the ANOVA effect sizes for the SLiD and SLi with arbitrary cutoff point methods. All methods presented a medium-to-large two-way interaction involving cross-loading size $\times$ number of factors $($ NUM.SF $\times$ CROSS.SD), implying that the negative effect of cross-loadings was reduced in small structures (e.g., c.c. decrease for NUM.SF = 3; SLi.05 = .12; c.c. decrease for NUM.SF = 6; SLi.05 = .23). The interaction effect size diminished as the cut-off for SLi increased (SLi.05 $\eta_{p}^{2}=.307$; SLi.10 $\eta_{p}^{2}=.290 ;$ SLi.15 $\eta_{p}^{2}=.251 ;$ SLi.20 $\left.\eta_{p}^{2}=.209\right)$, being SLiD the most robust method (SLiD $\eta_{p}^{2}=.066$ ). Lastly, no method was strongly affected by either sample size $\left(.007 \leq \eta_{p}^{2} \leq .020\right)$ or the number of variables per factor $\left(.003 \leq \eta_{p}^{2} \leq .098\right)$.

\section{[PLEASE INSERT TABLE 4 HERE]}

Previous results were concerned with the mean c.c. values for each complete structure (i.e., averaging over factors of different average loading size), which could provide a limited view of the performance of the rotation methods. When analysing the percentage of replicates that were satisfactorily recovered across conditions (Table 5, upper panel), SLiD performed better than SLi with any fixed cut-off when recovering all types of factors. The largest discrepancies were found for c.c. > .95 with factors composed of high loadings $(\bar{\lambda}=.60$; difference $\approx 40 \%)$. Nevertheless, results illustrated that not all factors were equally recovered, and that each factor appropriate cut-off was dependent upon factor loading magnitude. Regarding general, high and medium size 
factors, all arbitrary cut-offs performed similarly well (average c.c. difference $=.001$ ), where increasing the cut-off point somewhat improved the recovery for these factors.

However, the opposite effect was found when analysing the factors with low magnitude loadings. As the cut-off increased, the SLi performance was greatly diminished (c.c. differences: SLi.05 and SL.10 = .049; SLi.05 and SL.15 = .076; SLi.05 and SL. $20=.212$ ). It is noteworthy that even though SLiD still outperformed all SLi methods for factors that had low loadings, it still struggled to adequately recover these factors $($ SLiD low c.c. $=.829)$.

\section{[PLEASE INSERT TABLE 5 HERE]}

Despite the overall results, it should be noted that SLi with a fixed cut-off point provides a good recovery under optimal conditions (i.e., CROSS.SD = .05; Table 5, lower panel). All SLi methods with arbitrary cut-offs satisfactorily recovered the general, the high and medium loading size factors (i.e., all average c.c. $\geq .85$ ), benefitting again from applying higher cut-offs. Once more, SLi methods with arbitrary cut-offs failed to recover the low loadings factors, especially when applying higher cutoffs (SLi.15 c.c. low loadings $=.775 ;$ SLi.20 c.c. low loadings $=.641$ ).

\section{Discussion}

Almost 80 years after their appearance, bi-factor models have become a major research topic in the psychometric literature. This article presented, for the first time, evidence of the consequences of using arbitrary cut-off points for exploratory bi-factor target rotation. Furthermore, a new algorithm for the empirical estimation of accurate cut-offs under realistic conditions was introduced in this study. This new proposal was evaluated by means of an extensive Monte Carlo Simulation against alternative methods applying fixed cut-offs. 


\section{Main Findings}

The use of fixed cut-off points. Recommendations found in the EFA literature (i.e., .30 - .50; Izquierda, Olea \& Abad, 2014) were not satisfactorily translated into exploratory bi-factor analysis. Due to item variance partition specified in the bi-factor model, specific factor loadings could be expected to be lower than in other EFA applications, entailing the necessity of adapting factor saliency thresholds. According to our expectations, lower cut-offs benefited factor recovery when applying bi-factor target rotation. However, it was demonstrated that the impact of the arbitrary cut-off point was dependent upon the factor loading distribution, where higher cut-offs were significantly more affected by the presence of a factor with lower substantive loadings. Remarkably, factors with either high or medium sized loadings were recovered with similar accuracy by all fixed cut-offs evaluated and with a precision similar to previous studies (if similar conditions were evaluated; lower panel, Table 5). Furthermore, the inclusion of a low average factor loadings factor in combination with the presence of several crossloadings caused a substantial decrease in the overall accuracy for SLi with higher cutoff points (Table 5), particularly if compared with its performance reported in a previous study (Abad et al., 2017). Lastly, it should be acknowledged that, as also reported in earlier research, simple bi-factor structures, with none to a few low magnitude cross-loadings and high to medium average loadings factors, are expected to be correctly recovered by all methods, including SLi with high cut-off points (Table 2; Abad et al., 2017). Nevertheless, in realistic settings when structures are expected to either present specific factors with low average factor loadings, items with strong crossloadings, or its combination, SLiD should be preferred.

Empirical estimation of cut-off points. The proposed method based on loading differences (SLiD), which was used in conjunction with SLi rotation, was defined as an 
iterative, factor-specific, empirically estimated, automatic algorithm for defining partially specified target rotations based on an initial SL solution. SLiD objective is to find the first cross-loading in the sorted squared normalized factor loading distribution, and to fix values below such value in the target matrix. Additionally, and to o the authors' knowledge, this is the first target rotation algorithm deploying a criterion based on sorted, one-lagged differences obtained from the empirical factor loadings distribution.

Results evidenced that when compared to SLi with arbitrary cut-offs, this method improved factor recovery for all the studied conditions. Far from perfect, the performance of SLiD was greatly diminished under the presence of high size crossloadings, especially when recovering factors with low substantive loadings (Table 5). However, such a result may be due to the SLi algorithm itself (and not related with the method for defining the target matrix), as explained next.

The pitfall of the SLi algorithm. When inspecting replicates that were not adequately recovered, it was observed that factor collapse occurred in several cases, and that the higher the magnitude of these cross-loadings, the more aggravated the problem was. Indeed, factor collapse could be deemed as the consequence of a non-uniqueness problem of the simulated bi-factor structures (Green \& Yang, 2017). If by chance, or due the presence of cross-loadings (combined with the presence of factors differing in their average factor loadings), the reproduced correlation matrix presents a true dimensionality approximated to the one of a hierarchical solution (dimensionality equals the number of specific factors, not the number of specific factors + one as in the bi-factor case), SLi solutions could result in factor-collapsed solutions (which would be in accordance with the true dimensionality; Mansolf \& Reise, 2016). Additionally, these collapsed solutions could also be consistent with collapsed population, non-simulated, 
bi-factor structures that also approximate such reproduced correlation matrices.

Unfortunately, no trustworthy method for evaluating the uniqueness of a simulated bifactor structure or to preclude factor collapse on the basis of a simulated reproduced correlation matrix prior to factor rotation has been developed yet.

Furthermore, all SLi-based algorithms apply a partially specified target rotation where all general factor loadings, and only some of the specific factor loadings, are freed in the target matrix. This leads to the general factor to absorb as much variance as possible. Therefore, if researchers aim to obtain solutions not presenting collapse, applying an SL transformation would be a viable option in some cases (i.e., as the SL solution would provide a good fit to the data). Alternatively, unreported analyses confirmed that factor collapse could be prevented in some specific cases by applying a totally specified target rotation in SLiD. Such target matrix additionally fixes a value (typically \pm one) for expected substantive values in the target matrix (Browne, 2001), so that all elements in the target rotation are given a target value. However, there is no free lunch in target rotation: In a totally specified target rotation, two kinds of errors could simultaneously occur, as factor loadings are either being shrunk to zero or enlarged towards \pm one. The latter cannot occur in partially specified matrices, as the only distances to be minimized are those with respect to targets of zero. Unfortunately, the effect of applying each type of target rotation to bi-factor structures remains uninvestigated.

\section{Limitations and Future Directions}

Firstly, the current findings are limited to the conditions studied in the simulation, where the aim of the selected conditions was to reproduce realistic models covering a wide range of complex structures. Secondly, neither the impact of over and under factor 
extraction, nor items loading only in either the general or the specific factors were considered. Lastly, only bi-factor structures with orthogonal specific factors were simulated. Though alternative bi-factor structures with oblique specific-factors can be fitted, their interpretation is still a matter of controversy.

Interest in both bi-factor structures and target rotation methods have dramatically increased over the last decade. Today, the distinctions and equivalences between exploratory hierarchical and exploratory bi-factor structures have been substantively clarified (Mansolf \& Reise, 2016; Waller, 2017). Additionally, while the target rotation has become one of the most compelling alternatives for studying complex structures, its generalized application is still upheld by some unresolved limitations: a) how to identify the best number of elements to be fixed in the target matrix. The comparison of SLiD with alternative methods for objective empirical cut-off estimation (such as Promin; Lorenzo-Seva, 1999) shall be a priority of future research efforts; b) once the correct number of elements to be fixed in $\mathbf{B}$ is known, how to deal with optimal solutions prone to local minima problems (as occurs in Simplimax); and c) how to guarantee unique bifactor structures and identified rotated solutions. Whilst factor identification has received less attention than other topics in the literature, it should not be disregarded when applying target rotations.

Additionally, to ensure the predictive validity of an empirical procedure such as SLiD, a split-half cross-validation, similar to the one found in FACTOR (Ferrando \& Lorenzo-Seva, 2017), can be applied as follows²: a) firstly, divide the sample on two random subsets; b) Apply SLiD to the first sample to obtain an empirical, iteratively refined target matrix; c) Apply this target in a non-iterative fashion to an unrotated solution found in the second subset; d) If step b and c solutions are similar, researchers

\footnotetext{
${ }^{2} \mathrm{We}$ are indebted to an anonymous reviewer for suggesting this cross-validation procedure.
} 
can be more confident regarding the validity of the target applied by SLiD. Researchers should ensure, however, that both subsets are of sufficient sample size for both solutions to be properly estimated. Moreover, if researchers are interested in assessing the accuracy of such a procedure, a bootstrapping strategy could be of employed (Raykov \& Little; 1999).

Lastly, alternative methods based on target rotations have been recently proposed (BIFAD; Waller, 2017). However, the BIFAD method, which applies a totally specified target rotation, is also based on using arbitrary cut-off points. Future research should address how a loadings difference method for defining a totally specified target in the BIFAD algorithm would perform and compare it with the recommendations for defining arbitrary cut-off points, which the authors are currently investigating (p. 12; Waller, 2017). 


\section{Electronic Supplementary Material}

ESM 1. Supplementary_Code.R

This R Script file contains the SLiD algorithm code, as well as the necessary auxiliary functions to run the SLi function. 


\section{References}

Abad, F. J., Garcia-Garzon, E., Garrido, L. E., \& Barrada, J. R. (2017). Iteration of Partially Specified Target Matrices : Application to the Bi-Factor Case. Multivariate Behavioral Research, 52(4), 416-429. http://doi.org/10.1080/00273171.2017.1301244

Asparouhov, T., \& Muthén, B. (2009). Exploratory Structural Equation Modeling Exploratory Structural Equation Modeling. Structural Equation Modeling: A Multidisciplinary Journal, 16(3), 397-438.

http://doi.org/10.1080/10705510903008204

Bollmann, S., Heene, M., \& Küchenhoff, H. (2015). What can the Real World do for simulation studies? A comparison of exploratory methods. (Technical Report 181). Retrieved from Department of Statistics, University of Munich: https://epub.ub.uni-muenchen.de/24518/

Browne, M. W. (1972). Orthogonal Rotation to a Partially Specified Target. British Journal of Mathematical and Statistical Psychology, 25, 115-120. https://doi.org/10.1111/j.2044-8317.1972.tb00482.x

Browne, M. W. (2001). The Latent Structure of Memory : A Confirmatory FactorAnalytic Study of Memory Distinctions. Multivariate Behavioral Research, 36(1), 29-51. http://doi.org/10.1207/S15327906MBR3601

Chen, F.F., West, S.G., \& Sousa, K.H. (2006). A comparison of bi-factor and second order models of quality-of-life. Multivariate Behavioral Research, 41(2), 189-225. doi:10.1207/s15327906mbr4102_5

Cohen, J. (1988). Statistical power analysis for the behavioural sciences (2nd Editio). Hillsdale, N.J.: Lawrence Erlbaum. 
Ferrando, P. J., \& Lorenzo-Seva, U. (2017). Program FACTOR at 10: Origins, development and future directions. Psicothema, 29, 236-240. doi: 10.7334/psicothema2016.304

Genz, A., Bretz, F., Miwa, T., Mi, X., Leisch, F., Scheipl, F., Bornkamp, B., Maechler, M., \& Hothorn, T. (2017). mvtnorm: Multivariate Normal and t Distributions. R package version 1.0-6. URL: https://CRAN.R-project.org/package=mvtnorm.

Green, S., \& Yang, Y. (2017). Empirical Underidentification with the Bifactor Model: A Case Study. Educational and Psychological Measurement, 1-20. http://doi.org/10.1177/0013164417719947

Hattori, M., Zhang, G., \& Preacher, K. J. (2017). Multiple Local Solutions and Geomin Rotation. Multivariate Behavioral Research, 52(6), 720-731. http://doi.org/10.1080/00273171.2017.1361312

Holzinger, K. J., \& Swineford, F. (1937). The Bi-factor Method. Psychometrika, 2(1), 41-54. https://doi.org/10.1007/BF02287965

Izquierdo, I., Olea, J., \& Abad, F. J. (2014). Exploratory factor analysis in validation studies: Uses and recommendations. Psicothema, 26(3), 395-400. doi: 10.7334/psicothema2013.349

Jamovi project (2018). jamovi (Version 0.8.1.13) [Computer Software]. Retrieved from https://www.jamovi.org

Jennrich, R. I. (2004). Rotation to Simple Loadings Using Component Loss Functions: The Orthogonal Case. Psychometrika, 69(2), 257-273. doi: 10.1007/S11336-003$1136-\mathrm{B}$

Jennrich, R. I., \& Bentler, P. M. (2011). Exploratory Bi-Factor Analysis. Psychometrika, 76(4), 537-549. doi: 10.1007/s11336-011-9218-4.

Jennrich, R. I., \& Bentler, P. M. (2012). Exploratory Bi-factor Analysis: The Oblique 
Case. Psychometrika, 77(3), 442-454. doi: 10.1007/s11336-012-9269-1.

Kiers, H. A. L. (1994). Simplimax: oblique rotation to an optimal target with simple structure. Psychometrika, 59(4), 567-579. doi:10.1007/BF02294392

Lorenzo-seva, U. (1999). Promin : A Method for Oblique Factor Rotation Promin : A Method for Oblique Factor Rotation. Multivariate Behavioral Research, 34(3), 347-365. doi: 10.1207/S15327906MBR3403_3

Lorenzo-Seva, U., \& ten Berge, J. M. F. (2006). Tucker's Congruence Coefficient as a Meaningful Index of Factor Similarity. Methodology, 2(2), 57-64. doi: $10.1027 / 1614-2241.2 .2 .57$

Mansolf, M., \& Reise, S. P. (2016). Exploratory Bifactor Analysis : The SchmidLeiman Orthogonalization and Jennrich-Bentler Analytic Rotations. Multivariate Behavioral Research, 51(5), 698-717. doi : 10.1080/00273171.2016.1215898

Meade, A. W. (2008). Power of AFIs to Detect CFA Model Misfit. Paper presented at the 23th Annual Conference of the Society for Industrial and Organizational Psychology. San Francisco, CA.

Moore, T. M., Reise, S. P., Depaoli, S., \& Haviland, M. G. (2015). Iteration of Partially Specified Target Matrices: Applications in Exploratory and Bayesian Confirmatory Factor Analysis. Multivariate Behavioral Research, 50(2), 149-161. http://doi.org/10.1080/00273171.2014.973990

Morin, A. J. S., Arens, A. K., \& Marsh, H. W. (2015). A Bifactor Exploratory Structural Equation Modeling Framework for the Identification of Distinct Sources of Construct-Relevant Psychometric Multidimensionality. Structural Equation 
Modeling: A Multidisciplinary Journal, 23(1), 116-139. doi:10.1080/10705511.2014.961800

Myers, N. D., Ahn, S., \& Jin, Y. (2013). Rotation to a Partially Specified Target Matrix in Exploratory Factor Analysis: How Many Targets? Structural Equation Modeling: A Multidisciplinary Journal, 20(1), 131-147. doi:

$10.1080 / 10705511.2013 .742399$

Myers, N. D., Jin, Y., Ahn, S., Celimli, S., \& Zopluoglu, C. (2015). Rotation to a partially specified target matrix in exploratory factor analysis in practice. Behavior Research Methods, 47(2), 494-505. doi: 10.3758/s13428-014-0486-7

Peeters, C. F. W. (2012). Rotatitional Uniqueness Conditions Under Oblique Factor Correlation Metric. Psychometrika, 77(2), 288-292. doi : 10.1007/S11336-0129259-3

R Core Team (2017). R: A language and environment for statistical computing. $\mathrm{R}$ Foundation for Statistical Computing, Vienna, Austria. URL: https://www.Rproject.org/.

Raykov, T., \& Little, T. D. (1999). A Note on Procrustean Rotation in Exploratory Factor Analysis: A Computer Intensive Approach to Goodness-of-Fit Evaluation. Educational and Psychological Measurement, 59(1), 47-57. doi: $10.1177 / 00131649921969730$

Reise, S. P. (2012). The Rediscovery of Bifactor Measurement Models. Multivariate Behavioral Research, 47(5), 667-696. doi: 10.1080/00273171.2012.715555

Reise, S. P., Moore, T. M., \& Haviland, M. G. (2010). Bifactor Models and Rotations: Exploring the Extent to which Multidimensional Data Yield Univocal Scale Scores. Journal of Personality Assessment, 92(6), 544-559. doi: 
10.1080/00223891.2010.496477.Bifactor

Reise, S. P., Moore, T., \& Maydeu-Olivares, A. (2011). Target Rotations and Assessing the Impact of Model Violations on the Parameters of Unidimensional Item Response Theory Models. Educational and Psychological Measurement, 71(4), 684-711. doi: 10.1177/0013164410378690

Revelle, W. (2017). Psych: Procedures for Personality and Psychological Research, Northwestern University, Evanston, IL, USA. URL: https: //CRAN.Rproject.org/package $=$ psch. Version 1.7.8.

Schmid, J., \& Leiman, J.M. (1957). The development of hierarchical factor solutions. Psychometrika, 22 (1), 53-61. doi: 10.1007/BF02289209

Trendafilov, N. T. (1994). A Simple Method for Procrustean Rotation in Factor Analysis Using Majorization Theory. Multivariate Behavioral Research, 29 (4), 385-408. doi : 10.1207/s15327906mbr2904_4

Tucker, L. R. (1951). A method for synthesis of factor analysis studies (Personal Research Section Report No. 984). Washington, D.C: Department of the Army.

Waller, N. G. (2017). Direct Schmid-Leiman Transformations and Rank-Deficient Loadings Matrices. Psychometrika, 83 (4), 858-870. doi: 10.1007/s11336-0179599-0 


\section{Table 1.}

SLiD algorithm first iteration target matrix definition for Chen, West \& Souza (2006) data.

\begin{tabular}{|c|c|c|c|c|c|c|c|c|c|c|c|c|}
\hline \multicolumn{5}{|c|}{ Panel A: Original SL solution } & \multicolumn{4}{|c|}{ Panel B: Squared normalized loadings } & \multicolumn{4}{|c|}{ Panel E: First iteration target matrix } \\
\hline Item & SF1 & SF2 & SF3 & SF4 & SF1 & SF2 & SF3 & SF4 & SF1 & SF2 & SF3 & SF4 \\
\hline 1 & .67 & .00 & .01 & -.01 & 1.00 & .00 & .00 & .00 & NA & 0 & 0 & 0 \\
\hline 2 & .47 & .06 & -.01 & .04 & .98 & .02 & .00 & .01 & NA & 0 & 0 & 0 \\
\hline 3 & .64 & .01 & .01 & .02 & 1.00 & .00 & .00 & .00 & NA & 0 & 0 & 0 \\
\hline 4 & .67 & .00 & -.02 & -.03 & 1.00 & .00 & .00 & .00 & NA & 0 & 0 & 0 \\
\hline 5 & .63 & .00 & .04 & .00 & 1.00 & .00 & .00 & .00 & NA & 0 & 0 & 0 \\
\hline 6 & .02 & .54 & .04 & -.03 & .00 & .99 & .01 & .00 & 0 & NA & 0 & 0 \\
\hline 7 & -.01 & .38 & .04 & .07 & .00 & .96 & .01 & .03 & 0 & NA & 0 & 0 \\
\hline 8 & .06 & .57 & -.04 & .06 & .01 & .97 & .00 & .01 & 0 & NA & 0 & 0 \\
\hline 9 & -.03 & .39 & .15 & -.02 & .01 & .86 & .13 & .00 & 0 & NA & NA & 0 \\
\hline 10 & .02 & -.04 & .36 & .02 & .00 & .01 & .98 & .00 & 0 & 0 & NA & 0 \\
\hline 11 & .05 & .00 & .31 & .08 & .02 & .00 & .92 & .06 & 0 & 0 & NA & 0 \\
\hline 12 & -.03 & .06 & .33 & -.02 & .01 & .03 & .96 & .00 & 0 & 0 & NA & 0 \\
\hline 13 & .24 & .01 & .17 & .09 & .61 & .00 & .31 & .09 & NA & 0 & NA & 0 \\
\hline 14 & .13 & .05 & .27 & .01 & .18 & .03 & .79 & .00 & NA & 0 & NA & 0 \\
\hline 15 & .03 & -.02 & .00 & .60 & .00 & .00 & .00 & 1.00 & 0 & 0 & 0 & NA \\
\hline 16 & .00 & .13 & .02 & .44 & .00 & .08 & .00 & .92 & 0 & 0 & 0 & NA \\
\hline 17 & -.02 & .02 & .01 & .52 & .00 & .00 & .00 & 1.00 & 0 & 0 & 0 & NA \\
\hline Avera & .21 & .13 & .10 & .11 & .34 & .23 & .24 & .18 & & & & \\
\hline
\end{tabular}

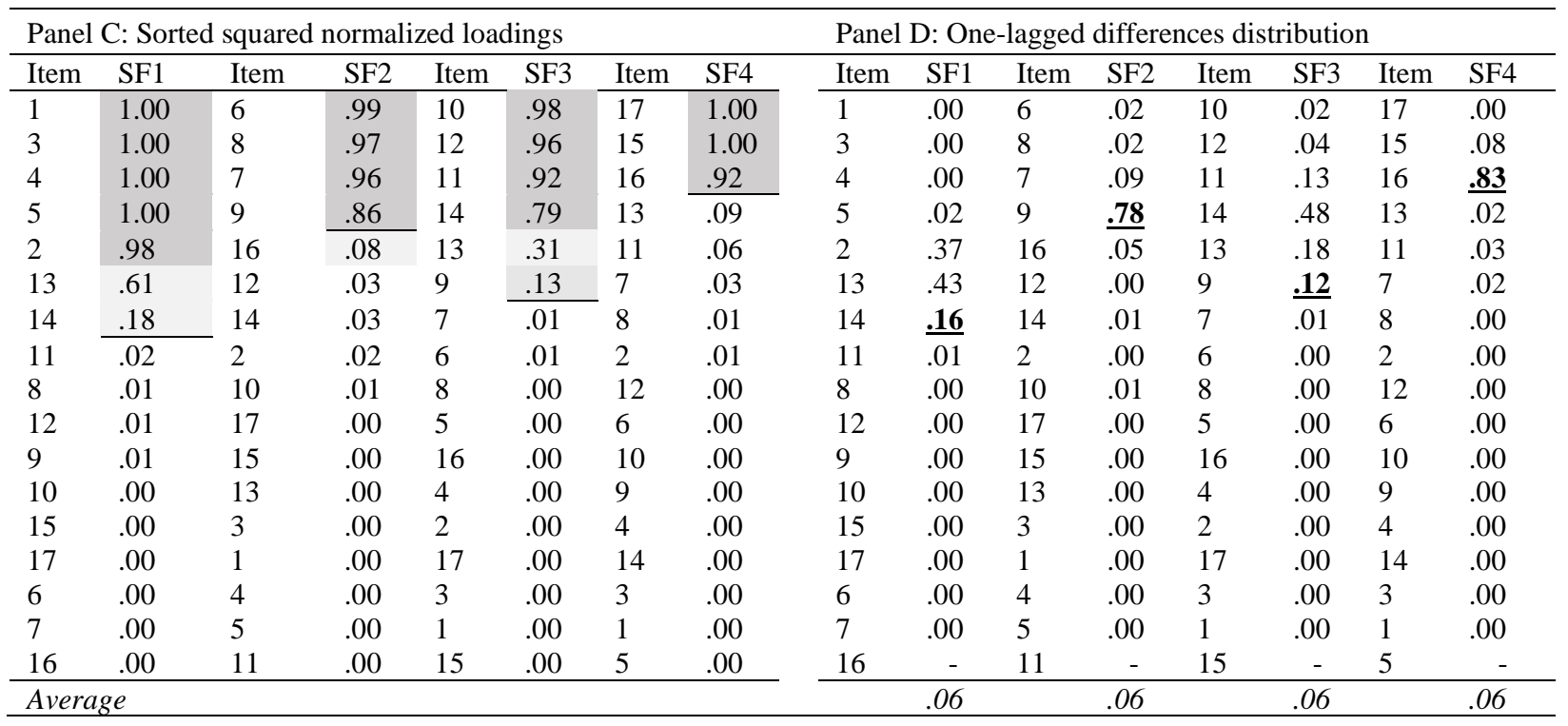

Note: $\mathrm{SF}=$ specific factor (SF1: Cognition; SF2: Vitality; SF3: Mental health; SF4: Disease worry); Panels divided as item order is changed between Panels B and C. In Panel A substantive loadings appear shadowed in strong grey and cross-loadings in light grey. First one-lagged difference above factor difference average is presented bolded and underlined. 
Table 2.

Manipulated factors included in the Monte Carlo study.

\begin{tabular}{ll}
\hline Factor & Levels \\
\hline Sample Size & Low $(\mathrm{n}=500) ;$ \\
$(\mathrm{N})$ & Medium $(\mathrm{n}=1000) ;$ \\
& High $(\mathrm{n}=2000)$ \\
Indicators per specific factor & Low $(4) ;$ \\
(VAR.SF) & Medium $(6) ;$ \\
& High (12) \\
Number of specific factors & Low (3); \\
(NUM.SF) & High $(6)$ \\
Cross-loading average size & Low $(\mathrm{M}=.00, \mathrm{SD}=.05) ;$ \\
(CROSS.SD) & Medium $(\mathrm{M}=.00, \mathrm{SD}=.10) ;$ \\
& High $(\mathrm{M}=.00, \mathrm{SD}=.15)$. \\
\hline
\end{tabular}


Table 3.

Marginal mean factor recovery for the SLi with arbitrary cut-off points and SLiD algorithms.

\begin{tabular}{|c|c|c|c|c|c|}
\hline Variable / Level & SLiD & SLi.05 & SLi.10 & SLi.15 & SLi.20 \\
\hline \multicolumn{6}{|l|}{$\underline{\mathrm{N}}$} \\
\hline 500 & .920 & .845 & 842 & .835 & .817 \\
\hline 1000 & .927 & .851 & 849 & .843 & .821 \\
\hline 2000 & .930 & .858 & 857 & .851 & .829 \\
\hline \multicolumn{6}{|l|}{ VAR.SF } \\
\hline Low (4) & .911 & .834 & .834 & .834 & .827 \\
\hline Medium (8) & .930 & .855 & .851 & 844 & .822 \\
\hline High (12) & .936 & .866 & .861 & .851 & .819 \\
\hline \multicolumn{6}{|l|}{ NUM.SF } \\
\hline Low (3) & .930 & .886 & .881 & .868 & .835 \\
\hline High (6) & .921 & .817 & 817 & .818 & .810 \\
\hline \multicolumn{6}{|l|}{ CROSS.SD } \\
\hline Low (.05) & .965 & .934 & 931 & 921 & 887 \\
\hline Medium (.10) & .932 & .858 & .855 & .851 & 831 \\
\hline High (.15) & .880 & .762 & .760 & .757 & .749 \\
\hline AVERAGE & .926 & .851 & .849 & .843 & .826 \\
\hline
\end{tabular}

Note. $\mathrm{N}$ = sample size; VAR.SF = variables per specific factor; NUM.SF = number of specific factors; CROSS.SD $=$ standard deviation for cross-loading simulation. Congruence coefficients $\geq .85$ appear shadowed in grey. Best c.c. for each condition appears bolded and underlined 
Table 4.

Univariate Analysis of Variance (ANOVA) effect sizes for SLi with arbitrary cut-off points and SLiD.

\begin{tabular}{lccccc}
\hline Effect Type / Variables & SLiD & SLi.05 & SLi.10 & SLi.15 & SLi.20 \\
\hline $\begin{array}{l}\text { Main Effects } \\
\text { N }\end{array}$ & .014 & .020 & .020 & .017 & .007 \\
$\quad$ VAR.SF & .095 & .098 & .061 & .018 & .003 \\
$\quad$ NUM.SF & .051 & .538 & .462 & .297 & .102 \\
$\quad$ CROSS.SD & .519 & .771 & .737 & .646 & .454 \\
Two Way Interactions & .066 & .307 & .290 & .251 & .209 \\
\hline$\quad$ NUM.SF $\times$ CROSS.SD & Note. N = sample size; VAR.SF $=$ number of variables per specific factor. NUM.SF $=$ number of specific factors; \\
CROSS.SD = cross-loading standard deviation. The dependent variable in the ANOVAs was the congruence \\
coefficient. Only medium $\left(\eta^{2}>.06\right)$ or larger effects are presented, with large $\left(\eta^{2}>.14\right)$ effects shadowed in grey.
\end{tabular}




\section{Running Head: BI-FACTOR ROTATION BASED ON LOADING DIFFERENCES}

\section{Table 5 .}

Proportion of replicates with mean c.c. higher than .85 and .95 for SLiD and SLi across all conditions (upper panel) and for low cross-loadings condition (lower panel).

\begin{tabular}{|c|c|c|c|c|c|c|c|c|c|c|c|c|c|c|c|}
\hline & \multicolumn{5}{|c|}{ Mean c.c. } & \multicolumn{5}{|c|}{ c.c. $>.85$} & \multicolumn{5}{|c|}{ c.c. $>.95$} \\
\hline \multicolumn{16}{|c|}{ All CROSS.SD conditions } \\
\hline Factor & SLiD & SLi.05 & SLi.10 & SLi.15 & SLi.20 & SLiD & SLi.05 & SLi.10 & SLi.15 & SLi.20 & SLiD & SLi.05 & SLi.10 & SLi.15 & SLi.20 \\
\hline General & .991 & .990 & .990 & .991 & .992 & $100 \%$ & $100 \%$ & $100 \%$ & $100 \%$ & 100 & $99 \%$ & $99 \%$ & $99 \%$ & $99 \%$ & $99 \%$ \\
\hline High & .966 & .893 & .896 & .898 & .900 & $99 \%$ & $76 \%$ & $77 \%$ & $79 \%$ & $79 \%$ & $82 \%$ & $42 \%$ & $45 \%$ & $47 \%$ & $47 \%$ \\
\hline Medium & .921 & .827 & $\underline{.828}$ & .827 & .824 & $89 \%$ & $53 \%$ & $54 \%$ & $55 \%$ & $55 \%$ & $47 \%$ & $17 \%$ & $18 \%$ & $19 \%$ & $19 \%$ \\
\hline Low & .829 & .740 & .691 & .664 & .528 & $54 \%$ & $28 \%$ & $27 \%$ & $25 \%$ & $18 \%$ & $13 \%$ & $3 \%$ & $3 \%$ & $1 \%$ & $1 \%$ \\
\hline \multicolumn{16}{|c|}{ CROSS.SD $=.05$} \\
\hline General & 1.00 & .998 & .999 & .998 & .998 & $100 \%$ & $100 \%$ & $100 \%$ & $100 \%$ & $100 \%$ & $100 \%$ & $100 \%$ & $100 \%$ & $100 \%$ & $100 \%$ \\
\hline High & .990 & .969 & .972 & .973 & .973 & $100 \%$ & $100 \%$ & $100 \%$ & $100 \%$ & $100 \%$ & $100 \%$ & $95 \%$ & $98 \%$ & $99 \%$ & $99 \%$ \\
\hline Medium & .970 & .936 & .939 & .940 & .940 & $98 \%$ & $97 \%$ & $97 \%$ & $98 \%$ & $97 \%$ & $95 \%$ & $44 \%$ & $48 \%$ & $49 \%$ & $50 \%$ \\
\hline Low & .901 & .836 & .812 & .775 & .641 & $88 \%$ & $63 \%$ & $62 \%$ & $57 \%$ & $40 \%$ & $31 \%$ & $8 \%$ & $7 \%$ & $7 \%$ & $7 \%$ \\
\hline
\end{tabular}

Note. SLiD: Schmid-Leiman Iterative Bi-factor Difference-based Target Rotation. In case of NUM.SF=6 structures, high, medium and low loadings factors represent the average of the two factors with such characteristics, respectively. Mean c.c. below .85 is presented bolded and underlined. Conditions with cells with less than $50 \%$ appear shadowed in grey. 